\title{
Network-Aware Genetic Algorithms for the Coordination of MALE UAV Networks
}

\author{
Alexandros Giagkos ${ }^{1}$, Myra S. Wilson ${ }^{2}$, and Ben Bancroft ${ }^{2}$ \\ 1 College of Engineering and Physical Sciences, Aston University, Birmingham, UK. \\ a.giagkos@aston.ac.uk \\ 2 Department of Computer Science, Aberystwyth University, Aberystwyth, UK. \\ mxw@aber.ac.uk, ben@benbancroft.uk
}

\begin{abstract}
Maintaining an ad hoc network infrastructure to cover multiple ground-based users can be achieved by autonomous groups of hydrocarbon powered medium-altitude, long-endurance (MALE) unmanned aerial vehicles (UAVs). This can be seen as an optimisation problem to maximise the number of users supported by a quality network while making efficient use of the available power. We present an architecture that combines genetic algorithms with a network simulator to evolve flying solutions for groups of UAVs. Results indicate that our system generates physical network topologies that are usable and offer consistent network quality. It offers a higher goodput than the non-networkaware equivalent when covering the communication demands of multiple ground-based users. Most importantly, the proposed architecture flies the UAVs at lower altitudes making sure that downstream links remain active throughout the duration of the mission.
\end{abstract}

Keywords: Genetic Algorithms · Wireless Communication · Unmanned Aerial Vehicles · Networks.

\section{Introduction}

It is broadly recognised that area coverage for communication services is a promising application domain for cooperative UAVs [1, 2]. Genetic algorithms (GAs) offer significant advances with most of the research concentrated on coordination, route finding, path planning and constraint management in multi-UAV systems 3 5. In most of these contributions, the communication network between the UAVs is assumed. Exemplar works include Carruthers et al., [6] where the authors proposed a GA-based collision-aware coordination system for UAV missions related to surveillance and searching in unknown areas, with the assumption that the communications are constantly available.

Very few researchers have addressed the quality of network coverage for largescale missions offered by groups of UAVs. Noticeable examples include Agogino et al., 7. who optimised power levels and antenna orientations using GAs to maximise area coverage for ground-based users.

In this paper, we address the problem of autonomous position coordination for communication UAVs using GAs, by providing and maintaining an efficient 
airborne network infrastructure capable of supporting the communication needs of users on the ground. Our design includes a network-aware evaluation method for evolving solutions, which incorporates communication links' validation via a network simulator that implements a complete TCP/IP protocol stack. Thus, two objectives are addressed: i) to maximise the number of users being covered based on the available power and ii) to maximise the number of active UAVto-UAVand UAV-to-users links being provided.

Section 2 introduces the problem with a scenario. The design of the GAs we employ is found in Section 3 , and in Section 4 , we discuss the system's architecture along with the network-aware evaluation method. The experimental methodology and results are found in Sections 5 and 6 respectively. Final remarks and future work are included in Section 7.

\section{Problem Description}

MALE fixed-wing UAVs are equipped with two radio antennae; i) one isotropic for the UAV-to-UAV transmissions, and ii) one horn-shaped able to transfer data to the ground-based users. They have limited power for the communication, denoted as $P_{\max }$ with which they have to provide as many communication links as possible. All users, including the UAVs, are equipped with a Global Positioning System (GPS) and periodically broadcast information about their current positions.

Communication links are treated independently. A transmission is considered successful when a UAV's transmitter can feed its antenna with enough power to satisfy the quality requirements. A link is considered of good quality if the ratio of the energy per bit of information $E_{b}$ to the thermal noise in $1 \mathrm{~Hz}$ bandwidth $N_{0}$ is maintained. Eq. 1 expresses the transmitting power $P_{t}$ required to cover a user at slant range $d$, as shown in Fig. 1a. For further details on computing slant range values, the reader is encouraged to consult Giagkos et al. 8.

$$
P_{t}=p \times\left(d^{2} R_{b} \frac{E_{b}}{N_{0}} \frac{1}{G_{r} G_{t}}\left(\frac{4 \pi f}{c}\right)^{2} T_{s y s} K\right)
$$

The higher the UAV flies, the greater its altitude $h$, the wider its conical footprint on the ground, and thus the greater the area covered. Similarly, the longer the slant range $d$ between the transmitter and the receiver, the higher the signal power required to support the communication. The slant angle $\alpha$ to a user is calculated by applying spherical trigonometry using the available GPS data that each network user is expected to broadcast at regular intervals. A user needs to lie within the footprint of at least one UAV to be part of the network.

Noise related to obstacles is represented by the use of the elevation angle $\gamma$. A link is achieved when $\gamma \geq \omega$, with $\omega=10^{\circ}$. Subsequently, if $\gamma<\omega$ then the factor $p$ in Eq. 1 is set to 0 , indicating that no power is dedicated to that specific link, and thus the user is not covered. The link is ultimately considered 


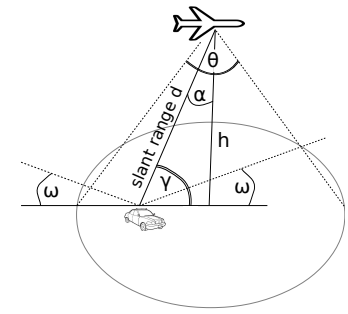

(a) Illustrating parameters to calculate slant range $d$ and factor $p$.

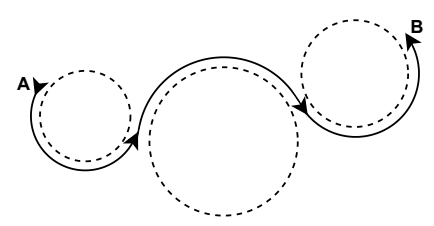

(b) Three segments of different duration $\delta h_{i}$ and bank angles $\beta_{i}$ between points $\mathrm{A}$ and $\mathrm{B}$.

Fig. 1: (a) A UAV's canonical footprint; (b) The Dubins path flying trajectory.

achievable if and only if $P_{t}$ is less than or equal to the remaining $P_{\max }$, the maximum power available for communications each cycle.

\section{Power-Aware Genetic Algorithms}

In our previous work, we describe how genetic algorithms can cooperatively relocate UAVs to maximise coverage [9. Flying trajectories are described by Dubins paths [10] consisting of 3 segments as depicted in Fig. 1b. We encode a UAV's trajectory as a 8-gene chromosome; three pairs of bank angle $\beta_{i}$ and associated duration $\delta t_{i}$ for each segment, with $i \in\{1,2,3\}$ and $\sum_{n=1}^{3}\left(\delta t_{i}\right)$ equal to the duration a complete trajectory. The final two genes are related to vertical flying with a binary $b$ indicating whether the altitude change $(\delta h)$ will be applied or the UAV will keep flying at constant altitude.

A single-point cross-over and a mutation operator are designed to evolve groups of $N$ number of flying trajectories for $N$ number of UAVs. A population of $M \times N$ are initiated, with $M=100$ number of groups. The best previous group is retained unchanged (i.e., elite), whereas all others are combined to form new offspring. Selection is performed using roulette wheel. Every two randomly selected chromosomes among chosen groups are reproduced with a probability of 0.3. Each offspring gene is mutated; we apply a random Gaussian offset (mean 0.0 , stdev. 0.1) to all real-valued genes, whereas the binary one is just flipped. The mutation rate is 0.05 . Finally, GAs run for 200 generations or until the allowed computation time has elapsed, with $M-1$ trajectories created at each generation. The time criterion is set to the time necessary to complete the default built-in circle manoeuvre when no solution is available.

The power-aware GAs utilise an evaluation method that measures the fitness of each group collectively. Given that the key objective is to maximise the number of supported users when limited power is available, the fitness score for a solution is calculated by $\mathfrak{f}=\frac{\sum_{n=1}^{|U|}\left|C_{n}\right|}{|G|}$, with $U$ the set of all UAVs, and $C_{n}$ the packing array of the $n^{\text {th }} \mathrm{UAV}$ and $G$ the set of all users on the ground. 
Calculating the packing arrays for each UAV plays an important role in the efficiency of the searching algorithm. The packing algorithm that assigns users to appropriate UAVs was initially introduced in Giagkos et al. [9] and thus, its details are omitted. Packing favours those users that are low-maintenance (closer to the centre of the footprint) and, in turn, maximises the total coverage.

\section{Network-Aware Genetic Algorithms}

We extend our work to form new network-aware GAs that not only consider link budgets but also evaluate each solution by measuring its network topology qualities. We integrate the system with NS3, a discrete-event network simulator capable of providing realistic network phenomena and monitoring network performance metrics [11. In this section, we describe the integration before documenting the internal mechanisms of the proposed network-aware GAs.

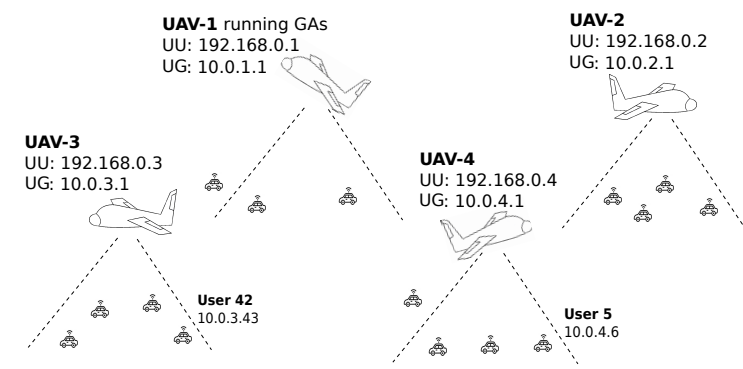

Fig. 2: Illustration of one NS3 network topology depicting a group of 4 UAVs. Each UAV gateway relays packets to and from its own footprint's network.

\subsection{Integration with a Network Simulator}

NS3's codebase is developed to allow the exchange of information between the GAs and the network simulator, namely the predicted positions of all users at the beginning of the next computational step, and the packing arrays. The builtin functionality to convert geographic to Cartesian coordinates is used, mapping the physical positions of all communicating users to a physical network topology.

All of the users' network interfaces are configured with respect to the packing information associated with each UAV, as seen in Fig. 2 Isotropic antennae's interfaces are given the address 192.168.0. $U_{n}$, with $U_{n}$ being the UAV's unique identifier (UID). Similarly, the horn-shaped antennae's addressed are set to $10.0 . U_{n} .1$ to facilitate footprint networks. Depending on which packing array it belongs to, each user receives an address $10.0 . U_{n} \cdot G_{j}$, with $G_{j}$ being its UID+1 and $U_{n}$ the UID of the supporting UAV. For example, user 42 in Fig. 2 uses the gateway address 10.0.3.1 to communicate with the rest of the network. 
NS3's wireless PHY and MAC implementations are based on the IEEE 802.11 standards. We increase the request to send / clear to send (RTS/CTS) timeout thresholds, overcoming the failed distributed coordination function pitfall in long-range transmissions. The gain of the horn-shaped antennae transmitters is set according to the manufacturer, i.e., calculated by $G_{t}=\frac{2 \eta}{1-\cos \left(\frac{\theta}{2}\right)}$, with $\theta=$ $125^{\circ}$ corresponding to the half-power beam-width angle of the antenna and $\eta=$ 0.95 , the efficiency of its transmission. For the isotropic antennae, gain $G_{t}=1$.

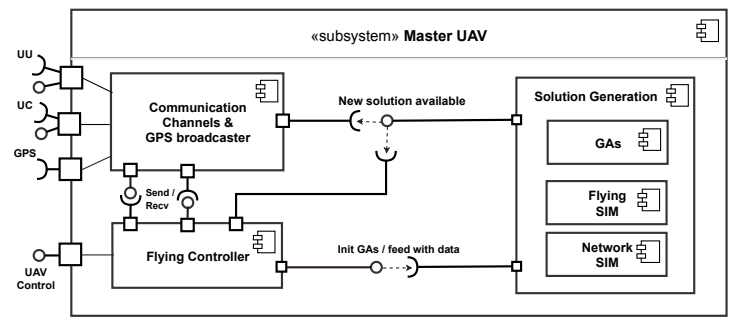

Fig. 3: Network-aware GAs architecture: the master UAV's internal components.

\subsection{Network Topology Evaluation Model}

The core differences between the power-aware and the network-aware GAs are the integration of the network simulator and its use to evaluate individual solutions by the latter GAs. The new objective is to maximise the fitness of each group of trajectories with respect to whether their resulting network topologies are able to maintain communication.

Fig. 3 depicts the internal components of the subsystem installed on the master UAV, which generates and distributes solutions to the rest of the group. The component responsible for the communication sends and receives data using the connected interfaces, including the GPS broadcaster. Data is ported to the flying controller, the component in charge of preparing the next steering parameters (i.e., bank angle and altitude changes). When no trajectory is available or incomplete, ${ }^{3}$ the controller initiates the GAs, feeding it at the same time with position related data.

Although the network-aware GAs share both encoding/decoding scheme and genetic operators with the power-aware GAs, the network-aware evaluation method also comprises short network trials (duration of 320 simulated seconds), designed to check the validity of all links of a solution's network topology. Link validation is a two-phase process and is performed by using a bespoke network protocol.

For UAV-to-UAV links, the master UAV broadcasts one discovery request packet per second to 192.168.0.255 containing a fresh sequence number (SeqNo). All receiving UAVs save the packet's SeqNo, update their routing tables and

\footnotetext{
${ }^{3}$ While the GAs search for solutions, the default UAV manoeuvre is to cruise in circles.
} 
broadcast the request further before acknowledging it by sending a unicast ACK packet to the master. Received broadcast packets that contain the same SeqNo are dropped, while unicast ACKs are forwarded using the routing tables. At the end of the trial, the UAV-to-UAV link validation metric is calculated as:

$$
N_{u u}=\frac{p k t_{a c k}}{p k t_{r e q} \times(|U|-1)}
$$

where $p k t_{a c k}$ is the number ACK packets received and $p k t_{r e q}$ the discovery requests sent to a topology of $|U|$ UAVs during the course of the trial.

Subsequently, UAV-to-ground links are checked by making use of the footprint networks. Every time a UAV acknowledges a discovery request, it broadcasts a request packet to its own network $\left(10.0 . U_{n} .255\right)$ with the same SeqNo. Any listening users acknowledge the request by sending ACK unicast packets. At every $t$ of the trial, a UAV-to-ground link validation metric $N_{u g}^{t}$ is given by:

$$
N_{u g}^{t}=\sum_{n=1}^{|U|}\left|C_{n}^{t, a c k}\right|, \quad \text { with } C_{n}^{t, a c k} \subseteq C_{n}^{t}
$$

where $C_{n}^{t}$ is the packing array for $U_{n}$ at $t$ and $C_{n}^{t, a c k}$ the subset of users from that array that have acknowledged support by $U_{n}$. Considering the whole duration $T$ of the trial, $N_{u g}$ is given by:

$$
N_{u g}=\frac{\sum_{t=1}^{T} N_{u g}^{t}}{\sum_{t=1}^{T}\left|\mathbb{C}^{t}\right|}, \quad \text { with } \mathbb{C}^{t}=\bigcup_{n=1}^{|U|} C_{n}^{t}
$$

with $\mathbb{C}^{t}$ the set of packed users at time $t$. Having calculated both $N_{u u}$ and $N_{u g}$, the fitness score for the evaluation of a group of $|U|$ flying trajectories is calculated as:

$$
\mathfrak{f}=w_{1} \times \frac{\sum_{n=1}^{|U|}\left|C_{n}\right|}{|G|}+w_{2} \times N_{u u}+w_{3} \times N_{u g}
$$

with $w_{1}, w_{2}$ and $w_{3}$ equally set to 0.33 for even contribution. Note that $C_{n}$ of each UAV is not expected to change significantly between every $t$ and $t+1$. Eq. 5 is designed to consider the packing algorithm that regulates the link budget, but also to examine whether the resulting network topology consists of active links for a sufficient period of time.

The fittest solution is decoded and returned to both the flying controller and the communication components, as shown in Fig. 3. At every next trial all routing tables are erased. Note that for the rest of the UAVs, the solution generation component is omitted from the architecture.

\section{Experimental Methodology}

To compare the power-aware with network-aware GAs groups of 4 UAVs are autonomously controlled to support 50 users uniformly distributed within a 100 
$\mathrm{km}^{2}$ terrain for 1 hour. All users follow a random waypoint mobility model, with varying speeds (5-60 mph) and pausing times of 120 seconds. The UAVs' speed is 75 knots and flying is constrained by a maximum bank angle of $48^{\circ}$ and altitude range of $150 \mathrm{~m}$ to $6 \mathrm{~km}$. Other parameters related to link budget are: $P_{\max }=50$ Watts, $\theta=125^{\circ}, \eta=0.95, R_{b}=2 \mathrm{Mbit} / \mathrm{s}, E_{b} / N_{0}=10$ $\mathrm{dB}$, frequency $f=5 \mathrm{GHz}$ and $\omega=10^{\circ}$.

The Ad-hoc On-demand Distance Vector (AODV) protocol [12] is used for routing. AODV offers a state-of-the-art reactive mechanism for discovering paths between sources and destinations. To ensure that users can only communicate within the assigned footprint, AODV is disabled outside their 10.0. $U_{n} .0$ networks.

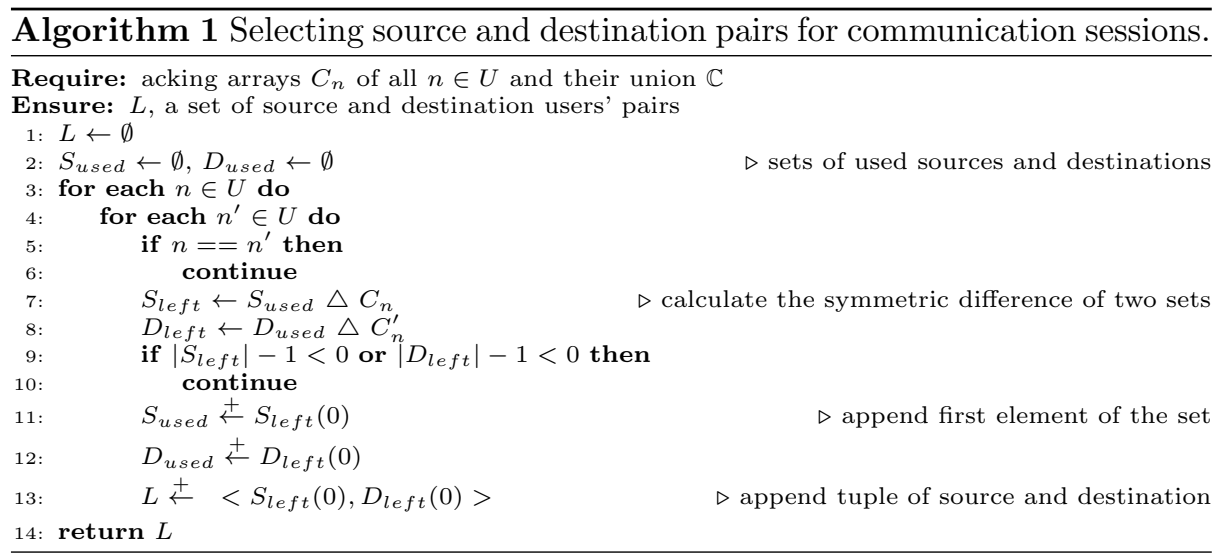

A $|U|(|U|-1)$ number of communication sessions are used in each experiment, with the selection of sources and destinations being performed using Algorithm 1. This approach ensures that the airborne backbone is fully utilised by the users on the ground. Constant Bit Rate (CBR) traffic generators are used for the communications between selected pairs, with sources transmitting 512-byte UDP datagrams at a rate of 1 Mbps.

Three performance metrics are used for the comparison of the two systems. Namely, i) coverage as the total number of supported users calculated by the packing algorithm, ii) goodput as the overall CBR throughput of the communication sessions excluding any protocol overhead bytes, and iii) altitude changes as the mean to examine the vertical activity of the group. The metrics highlight coverage capabilities with respect to both the number of users able to participate in the provided networks and the latter's efficiency in supporting communication services. Also, monitoring altitudes is important because it affects the footprint sizes and the slant range distances. As such, it offers useful insights about flying, revealing any emergent specialisation strategy.

Due to the stochastic nature of both systems, we repeat the experiments 30 times using random seeds and aggregate the results. 


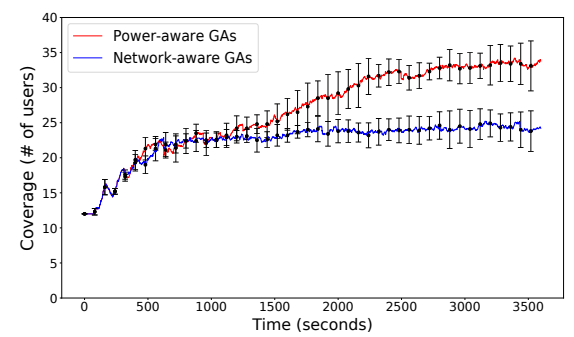

Fig. 4: Coverage results by power-aware and network-aware GAs.

\section{Results}

Fig. 4 shows coverage for both systems. Considering link validation causes a reduced number of supported users for the network-aware GAs. This is an expected result, the magnitude of which highlights the importance of considering network-related qualities. We observe that the network-aware system reaches a plateau while the power-aware exploits several windows of opportunity to improve coverage during the flight. This is due to the less conservative flying of the power-aware GAs, restricted only by the $P_{\max }$ values. This is justified by the altitude changes depicted in Fig. 6, where 3 out of 4 UAVs are found to almost reach the maximum altitude of $6 \mathrm{~km}$.

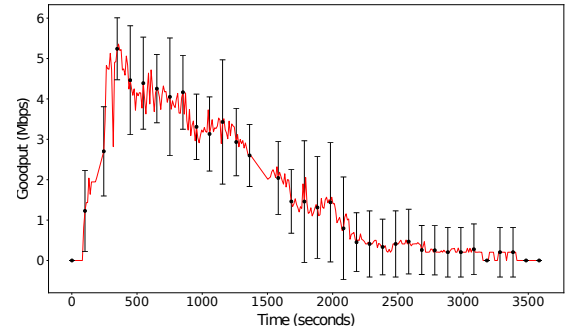

(a) Power-aware GAs.

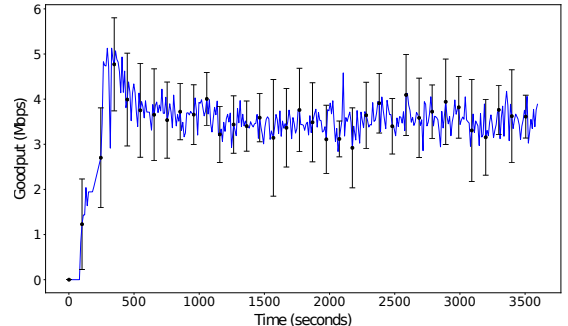

(b) Network-aware GAs.

Fig. 5: (a) Goodput results for both systems.

In practice, the network topologies generated by the two systems differ significantly in performance. Fig. 5 shows the goodput of the multiple CBR traffic generators. The network-aware GAs provide a usable and consistent infrastructure, whereas the links in the power-aware system gradually decline due to the lack of real network awareness of the power-aware GAs. For the latter, the distances between UAVs and their altitudes cause links to fail. Closely looking at the traffic data, we observe that communication is only possible for a single pair of users at the end of the experiment. 
Altitude changes caused by the network-aware GAs are found to be less frequent, as shown in Fig. 7. All UAVs fly level (approx. 4 km), ensuring that downstream links remain active. Specialisation in flying emerges less frequently than by the power-aware GAs, as activity in the vertical axis is significantly lower. Although the power-aware GAs seemingly put more effort in increasing coverage by changing their vertical formation, the resulting physical topologies are not efficient throughout the mission, mainly due to the internal mechanisms of the underlying network protocols combined with long-range transmissions. This is addressed by the network-aware GAs using the integrated evaluation method.

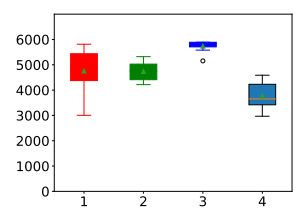

(a) $T=1500$

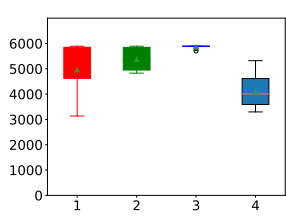

(b) $T=2000$

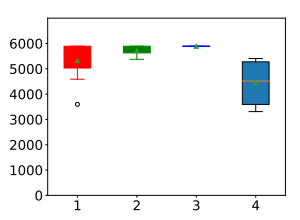

(c) $T=2500$

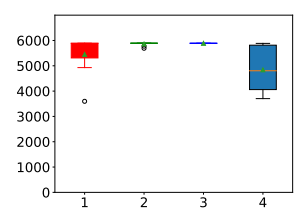

(d) $T=3000$

Fig. 6: Altitude in metres (y-axes) of 4 UAVs (x-axes) by power-aware GAs.

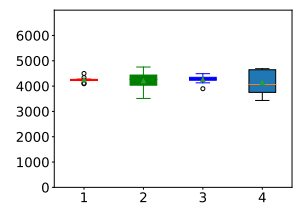

(a) $T=1500$

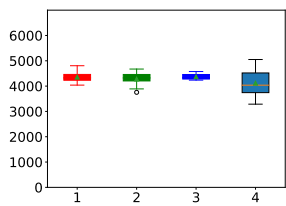

(b) $T=2000$

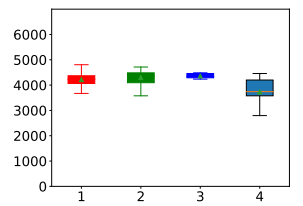

(c) $T=2500$

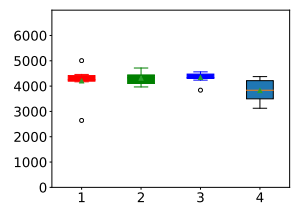

(d) $T=3000$

Fig. 7: Altitude in metres (y-axes) of 4 UAVs (x-axes) by network-aware GAs.

\section{Conclusions}

We presented a network-aware coordination system for MALE UAVs, which employs GAs to evolve flying solutions that result in effective physical topology networks. We discussed the integration with NS3 and compared the system's performance with a power-aware alternative that is purely based on link budget calculations. The integrated design offers a usable, consistent airborne infrastructure to support multiple users' communication demands. Considering the network-aware objective in the evaluation mechanism, via NS3, ensures that downstream links remain active throughout the mission and offer higher goodput than the power-aware GAs.

We deem the examination of the network-aware GAs under various scenarios as important future work to identify potential scalability issues. Consequently, we will investigate improvements to the evaluation method, such as considering application-related traffic to improve the performance of the overall network. 


\section{References}

[1] Navid Ali Khan, NZ Jhanjhi, Sarfraz Nawaz Brohi, Raja Sher Afgun Usmani, and Anand Nayyar. Smart traffic monitoring system using unmanned aerial vehicles (UAVs). Computer Communications, 157:434-443, 2020.

[2] Yong Zeng, Rui Zhang, and Teng Joon Lim. Wireless communications with unmanned aerial vehicles: opportunities and challenges. IEEE Communications Magazine, 54(5):36-42, 2016.

[3] Xiangyin Zhang and Haibin Duan. An improved constrained differential evolution algorithm for unmanned aerial vehicle global route planning. $A p$ plied Soft Computing, 26:270-284, 2015.

[4] Ferit Çakıcı, Halit Ergezer, Ufuk Irmak, and M Kemal Leblebicioğlu. Coordinated guidance for multiple UAVs. Transactions of the Institute of Measurement and Control, 38(5):593-601, 2016.

[5] D. Rathbun, S. Kragelund, A. Pongpunwattana, and B. Capozzi. An evolution based path planning algorithm for autonomous motion of a UAV through uncertain environments. In Digital Avionics Systems Conference, 2002. Proceedings. The 21st, volume 2, pages 8D2-1. IEEE, 2002.

[6] B. Carruthers, E. W. McGookin, and D. J. Murray-Smith. Adaptive evolutionary search algorithm with obstacle avoidance for multiple UAVs. In Pavel Zítek, editor, Proc. 16 ${ }^{\text {th }}$ IFAC World Congress, 2005, pages 20842084. International Federation of Automatic Control, 2005.

[7] Adrian Agogino, Chris HolmesParker, and Kagan Tumer. Evolving large scale UAV communication system. In Proceedings of the Fourteenth International Conference on Genetic and Evolutionary Computation Conference, GECCO '12, pages 1023-1030, New York, NY, USA, 2012. ACM.

[8] Alexandros Giagkos, Elio Tuci, Myra S. Wilson, and Philip B. Charlesworth. Evolutionary coordination system for fixed-wing communications unmanned aerial vehicles: supplementary online materials. http://www.aber.ac.uk/en/cs/research/ir/projects/nevocab, May 2021.

[9] Alexandros Giagkos, Elio Tuci, Myra S Wilson, and Philip B Charlesworth. UAV flight coordination for communication networks: genetic algorithms versus game theory. Soft Computing, pages 1-21, 2021. https://doi.org/10.1007/s00500-021-05863-6.

[10] Lester E. Dubins. On plane curves with curvature. Pacific Journal of Mathematics, 11(2):471-481, 1961.

[11] George F. Riley and Thomas R. Henderson. The ns-3 Network Simulator, pages 15-34. Springer Berlin Heidelberg, Berlin, Heidelberg, 2010.

[12] Charles Perkins, Elizabeth Belding-Royer, and Samir Das. RFC3561: Ad hoc on-demand distance vector (AODV) routing, 2003. 